\title{
E-Book as a Platform for Exploratory Learning Interactions
}

\author{
$\underline{\text { http://dx.doi.org/10.3991/ijet.v11i01.5011 }}$ \\ L.E. Nugroho \\ Universitas Gadjah Mada, Yogyakarta, Indonesia
}

\begin{abstract}
E-books are widely used as an important resource in learning. The most common way to use e-books is to incorporate them while a learning interaction is in progress. However, some learning styles are better supported inversely: e-books take the lead and are used to initiate and bind learning communications to the topics in focus. Unfortunately, existing e-book formats cannot accommodate this need effectively, potentially causing learning interactions to lose grip on the intended discussion framework. Inability to bind a conversation with the materials in the first place can also introduce overhead cost to the readers due to the burden of bringing the materials into the discussion.
\end{abstract}

In this work, we add the capability of e-books to serve as an interaction platform. An e-book is considered as a collection of objects indicating topics of learning discussions. Asynchronous conversation is anchored to one or more topic, and defined by a context that specifies participants and communications among them. Communication is modeled after a reactive mechanism by using event-action pairs. The proposed framework also accommodates dynamics within discussions, as well as structured discussions commonly found in many learning environments.

Index Terms-asynchronous discussions, learning, e-books, reactive systems.

\section{INTRODUCTION}

E-books are now widely accepted as one of the primary learning resources. A typical use of e-books is to incorporate them in a learning environment. During learning process, students can find an appropriate e-book, search for specific chapters, sections, or even paragraphs, and carry out knowledge acquisition process supported by descriptive materials in the e-book. While this becomes the most common way to use e-books, it may not be suitable for exploratory learning where students carry out the process in their own way without support of descriptive learning information $[1,2]$. Instead, students are offered an open learning environment where they have freedom to control what to read, what to try, which learning path to take, etc. In this mode of learning, the early phase is critical since students may not have any idea on where to start the exploration from and which direction to take. Discussions with fellow students and teachers can help, since learning can be more productive if carried out socially instead of in solitaire mode [3]. Furthermore, a few reading materials can be used to set the initial state of the discussions. The critical issue in this situation is how to bind a discussion to the materials, since the binding can keep students' mental state stay within the intended discussion framework, making exploratory process more effective.
E-book contents are good sources of materials, however, existing e-book formats are designed for documents for reading, therefore e-books can only wait passively for readers to pick them up and bring them into discussion sessions. While some formats such as epub allow limited interactions with readers (such as making annotations and highlighting texts) [4], it is currently not possible to explicitly bind student conversations to e-book contents and set a framework for the discussion. In effect, despite the availability of e-books, students' mental state cannot be secured to stay within the intended framework. As a result, conversations can move away from the intended course and students can be distracted from their exploration process.

The absence of initial materials in the early phase of exploratory learning through discussions would also incur overhead cost to students. Finding or selecting appropriate e-books, search for relevant materials inside, and bringing them into discussion require time and effort from students, which will be better allocated to the learning process itself.

To address the abovementioned problems, we propose a novel approach to integrate e-books with learning interactions that use it. The integration is achieved by linking a discussion to a set of topics contained in an e-book. One or more topics must be set up before any discussion on them can start. Binding a discussion to topics will build initial awareness and strong cohesion to keep the discussion within the intended framework, therefore minimizing the risk of distraction and increasing the effectiveness of knowledge acquisition during the discussion. This paper describes the underlying model of the interaction platform for e-books.

\section{PREVIOUS WORK}

While e-books are normally treated as a single entity where operations (e.g., uploading and reading) are performed to an e-book as a single object, some learning interactions need to dig into details and work with more detailed objects. For example, a focused discussion on a specific topic will make use of chapters, sections, or even paragraphs of an e-book, instead of the e-book as a whole. In this situation, the issues of representing, combining, and reusing learning objects will arise [5].

Naturally, learning interactions may involve many learning objects from different sources. In this situation, heterogeneity is a predominant factor, and to handle this situation, representation standards such as Shareable Content Object Reference Model (SCORM) [6] and Learning Object Metadata (LOM) [7] are developed. While allowing users to work with learning objects in a more flexible 


\section{SHORT PAPER}

\section{E-BOOK AS A PLATFORM FOR EXPLORATORY LEARNING INTERACTIONS}

way, these standards do not say anything about the binding between the users and the objects.

Research on integrating e-books with a learning environment takes different approaches. A visual approach is taken by Liang-Yi, et al. They provided e-books with visual cue map in the form of pages and within-page spatial cues in an interactive toolbar [8]. E-books can be equipped with flags, bookmarks, and comments presented visually, assisting readers to construct their cognitive map about the topic. A haptic-based approach is taken by Mahfujur Rahman, et al. [9]. They developed a framework for hapto-audio-visual-interaction with traditional reading materials used in a remote storytelling environment. A haptic e-book system can create remote storytelling sessions embedded with emotion expressions in the form of patterned vibrations and multimedia objects. Also in the visualization streamline, Kaplan and Chisik used e-book as a platform for collaborative learning [10]. They developed a system that can be used to bind user interactions and an e-book through the use of annotations created by "stamps" and "sticky notes".

\section{E-BOOK AS A COMMUNICATION PlatForm}

The aim of this research is to extend e-books with capabilities to handle discussions where learning interactions can be carried out. To do so, we have to redefine the structure of e-books. Instead of treating an e-book as a single lump of texts and images, we consider an e-book as a collection of objects. Objects can be any type (e.g., text, paragraphs, sections, chapters, tables, and images). They are used to represent topics of discussions.

Every discussion is bound to one or more objects. The relation is formed by linking context, a bounded conceptual area that "owns" a collection of interrelating entities, to objects that represent topics in focus. A context is constructed by a set of actors, their communications, and other actions that represent the dynamics of a discussion. The resulting "triangle" relation of objects, actors, and communications forms a cohesive construct of learning environment. Context binding must be prepared before a discussion can start.

We adopt asynchronous communication mode commonly used in popular social media such as Facebook and Twitter. Following this, conversations can be initiated by a triggering message (e.g., like status in Facebook or twit in Twitter). An initial message will spawn zero or more responding messages (e.g., comments to a status). There is no ordering and no moderation of those messages. During a discussion, new participants or topics can be added, or conversely they can be removed too.

\section{DESCRIPTION OF THE MODEL}

We describe our model formally as follows. An e-book $E$ is a collection of objects representing topics.

$$
\left\{T_{i}\right\} \in E
$$

A context $C$ is a bounded conceptual area and serves as a unit of discussion activities. A context consists of zero or more actors $A$ and zero or more operations $O$. Actors are objects representing participants in a discussion on one or more topics. Operations are capabilities owned by actors. By specifying actors and operations, we can define

relationships between actors. A context is always attached to a topic (we will explain it later).

$$
C_{i}:\left\{\left\{A_{i}\right\} \wedge\left\{O_{i}\right\}\right\}
$$

We then define the set of operations applicable to a context and its elements.

$$
\text { op : \{send; addT; rmvT; addA; rmvA; null }\}
$$

As the name implies, the send operation transmits a message $P$ from the sending actor $A_{i}$ to the receiving actor $A_{j}$.

$$
\operatorname{send}\left(A_{i} ; A_{j} ; P\right)
$$

The send operation mimics a comment in Facebook or a twit in Twitter that tags or mentions a particular target user. For a general comment (i.e., one that does not have a specific recipient), a "don't care" (*) symbol is used in the place of $A_{j}$.

The other operations, addT, $r m v T$, addA, and $r m v A$, are for adding and removing topics and actors, respectively.

$$
\begin{aligned}
& \operatorname{addT}\left(T_{1}, T_{2}, \ldots, T_{j}\right) \\
& \operatorname{rmv} T\left(T_{1}, T_{2}, \ldots, T_{j}\right) \\
& \operatorname{add} A\left(A_{1}, A_{2}, \ldots, A_{j}\right) \\
& \operatorname{rmv} A\left(A_{1}, A_{2}, \ldots, A_{j}\right)
\end{aligned}
$$

As previously mentioned, a discussion must be bound to a specific topic. To do this, one or more anchor topic must be brought into focus first. This is done through tagging. Once it is alive, we can attach a context to define the scope of discussion.

$$
\operatorname{tag}\left(T_{1}, T_{2}, \ldots, T_{j}\right): C_{i}
$$

A tag links one or more topic to a discussion environment. A topic becomes the starting point of a discussion. It is attached to the context that defines the participants and the interactions among them. A typical discussion environment can be set as follows.

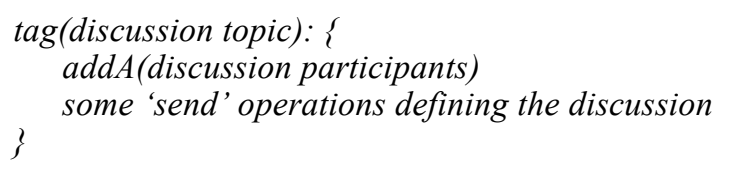

Tagging can be nested. In order to do this, a child tag must be defined within the scope of a context associated to the parent tag. The general rule of scoping applies here: the inner tag has narrower scope than the outer one. In practice, this structure can be used to model a discussion that moves towards a more focused topic.

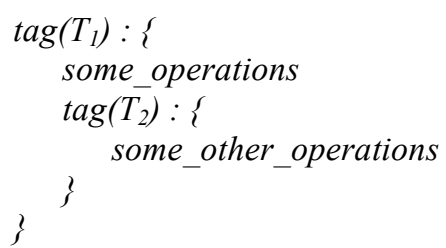




\section{SHORT PAPER}

\section{E-BOOK AS A PLATFORM FOR EXPLORATORY LEARNING INTERACTIONS}

Finally to make a context operational, we introduce a reactive mechanism to bind topics, actors, and operations in a single framework. The mechanism is based on events, and we employ the following rules on events: 1) an event can be system event or user-defined event, 2) a system event is generated by an operation, except null, while a user-defined event is triggered by a specific user status, and 3) the execution of any operation is always triggered by an event. A null operation is one that does nothing. It is used to define to end a conversation. The rules are shown in the following expressions, respectively.

$$
\begin{gathered}
\text { op } \rightarrow e \\
A[\text { status] } \rightarrow e \\
\text { on } e\{\text { op }\}
\end{gathered}
$$

\section{USING THE MODEL}

Given the event-based mechanism, it is now possible to define different types of discussions within the scope of a context. For example, a common Facebook-like discussion can be expressed as follows.

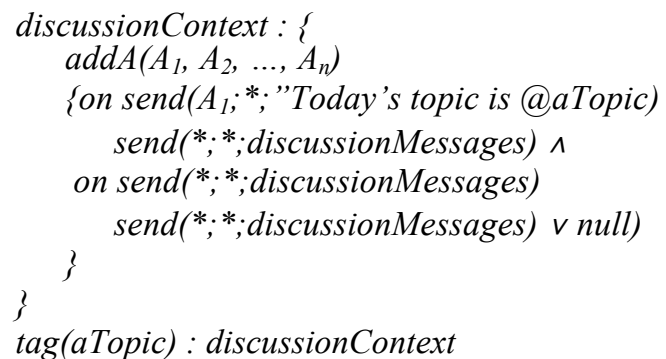

The first on statement initiates the discussion. The first message triggers an event that launches another message that responds the first message. The second on statement models the subsequent conversation. The conversation is described as interchanging messages originated from arbitrary actors. It terminates when a message has no further response, indicated by a null action.

Dynamic discussion can also be facilitated by adding or removing topics or participants. To do this, it is necessary to define an event that triggers the adding or removing operations. User-defined events can be flexibly used to express any situation that leads to new actions. As an example, a discussion can expand to include new topics when all participants agree to do so.

\section{on (A ['agree'], $A_{2}$ ['agree'], $A_{3}$ ['agree']) $\operatorname{add} T\left(T_{1}\right)$}

Our model can also be used to describe more structured interactions. For example, consider a class of 15 students, 3 mentors, and 1 teacher. The teacher sets up the class into 3 groups, each consists of 5 students and 1 mentor. Each group is assigned to discuss a chapter in a book. In the end of the group discussion, there is a summary session attended by all students and the teacher.

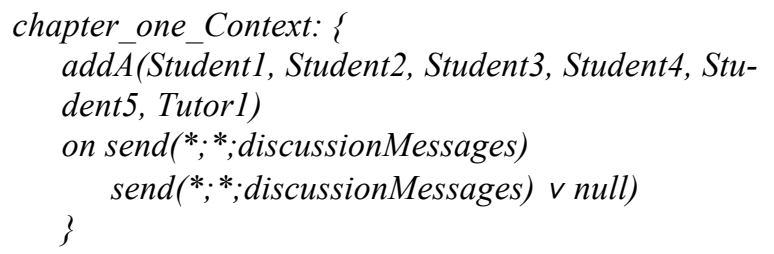

chapter_two_Context: addA'(Student6, Student7, Student8, Student9, Student10, Tutor2)

on send (*;*;discussionMessages) send $(* ; *$;iscussionMessages) v null) \}

chapter_three_Context: \{ addA (Student11, Student12, Student13, Student14, Student15, Tutor3)

on send (*;*;discussionMessages) $\operatorname{send}(* ; * ;$ discussionMessages) $v$ null) \}

summary_Context: \{Student1..Student15, Teacher; addA(Student1, Student2, Student3, Student4, Student5, Student6, Student7, Student8,

Student9, Student10, Student11, Student12, Student13, Student14, Student15, Teacher)

on send $(* ; * ;$ discussionMessages)

$\operatorname{send}(* ; *$;iscussionMessages) $v$ null) \}

$\operatorname{tag}($ Book $):\{$

tag(Chapter1) : \{chapter_one_Context\}

tag(Chapter2) : \{chapter_two_Context\}

tag(Chapter3) : \{chapter three Context\}

on allGroupDiscussionCompleted send(Teacher; *;summaryMessages) $\wedge$

on send $(* ; * ;$ discussionMessages) on send $(* ; *$;iscussionMessages) v null \}

\section{DISCUSSION}

The difference between this research and previous work described in $[8,9,10]$ is that their research treat e-book as an object in focus, and build interaction mechanism around it through artificial artifacts (e.g., stamps, sticky notes, or vibrations on specific parts of the e-book). This approach does not provide sufficient space for topic expansion (for example, to build a more holistic knowledge or understanding) since the nature of such artificial artifacts only provide limited scope of interactions (e.g., a vibration is used to mark a topic of interest, or a sticky note is only used to write short and focused expressions of interest. This is a serious limitation because the nature of exploratory learning requires unbounded scope of discussions.

On the other hand, our approach provides more flexibility to define the scope of an interaction. In contrast to the other methods, a tag in our model does not carry interaction messages. It is not used as a media of discussion, instead it only provides a context with a reference to a topic. Conversations are carried out within a context, and there is no limitation on their scope. With the capability of handling discussion dynamics (e.g., adding topics and participants) and complexity (e.g., structured discussions), our approach can easily accommodate various exploration requirements.

Our model does not adhere to a specific implementation strategy. We do not impose any restriction on how context, its binding with a topic, and event-based actions are implemented. Crucial issues in implementing our model are as follows. 
1. A context defines an abstract boundary. It defines all actors and their conversations in a specific discussion. In real implementation, it is important to make sure that all actors within a context have been enlisted before they can start any communication.

2. A similar situation also applies to the binding between a topic object and a context. All topic objects must be registered/defined within the realm of a context, and no unregistered/undefined object is allowed to be used in that context.

3. When a violation to the model occurs, the system should be able to respond accordingly. However, system behaviour upon a violation is defined by the programmer. A "hard" behaviour can be implemented by letting the system to respond with predefined actions, such as stopping the discussion at the point of violation. A "soft" behaviour may only produce warnings but high-level interactions can still continue.

4. The on construct requires a reactive mechanism. An implementation system should be able to work with event handling: capturing events and triggering specific actions.

\section{CONCLUSIONS}

We have presented a framework for exploratory learning interactions based on e-books. Different from other approaches that treat e-books as loose learning resources, we make components of an e-book as a platform for discussion, thereby binding the discussion to its topic. By linking to one or more topic, a discussion can be made consistent within its intended framework while maintaining the freedom to carry out topic expansion. Our approach also reduces overhead for participants, in order to discuss a specific topic they do not have to find the resources outside.

A simple asynchronous, non-moderated style of discussion is adopted to minimize the learning curve and maximize the eagerness of users to use this model. Gaining big user base certainly becomes an important goal, since it will open up larger pool of knowledge that can be accessed and shared in exploratory learning process.

\section{REFERENCES}

[1] L.P. Rieber, "Microworlds", in Handbook of Research for Educational Communications and Technology, Second edition, D. H. Jonassen, Ed., Simon and Schuster, 1996, pp. 583-603.

[2] M. Njoo, and T. De Jong, "Exploratory learning with a computer simulation for control theory: learning process and instructional support", Journal of Research in Science Teaching, vol. 30, no. 8, 1993, pp. 821-844. http://dx.doi.org/10.1002/tea.3660300803

[3] T. H. Cairney, (2003) "Literacy within family life", in Handbook of Early Childhood Literacy, N. Hall, J. Larson, and J. Marsh, Eds., London: Sage, 2003, pp. 85-98. http://dx.doi.org/10.4135/9781848608207.n8

[4] M. Gylling, W. McCoy, and M. Garrish, Eds., EPUB Publications 3.0.1, Recommended Specification, International Digital Publishing Forum. 28 June 2014, [Online]. Available: http://www.idpf.org/epub/301/ spec/epub-publications.html.

[5] A. Bouzeghoub, B. Defude, S. Arnrnour, J-F. Duitama, and C. Lecocq, "A RDF description model for manipulating learning objects", in Proceedings of IEEE International Conference on Advanced Learning Technology, 2004, pp. 81-85.

[6] C. Ostyn, "In the eye of the SCORM: an introduction to SCORM 2004 for content developers", 2007.

[7] IEEE, "1484.12.1-2002 - IEEE Standard for Learning Object Metadata", 2009.

[8] L. Liang-Yi, C. Gwo-Dong, and Y. Sheng-Jie, "Construction of cognitive maps to improve e-book reading and navigation", The Journal of Computers \& Education, vol. 60, Jan. 2013, pp. 32-39.

[9] A.S.M. Mahfujur Rahman, K.M. Alam, and A. El Saddik, "A prototype haptic e-book system to support immersive remote reading in a smart space", in IEEE International Workshop on Haptic Audio Visual Environments and Games (HAVE), 2011, pp. 124-128. http://dx.doi.org/10.1109/have.2011.6088405

[10] N. Kaplan, and Y. Chisik, "Reading alone together: creating sociable digital library books", in Proceedings of 2005 Conference on Interactive Design and Children, 2005, pp 88-94. http://dx.doi.org/10.1145/1109540.1109552

\section{AUTHOR}

L.E. Nugroho is with the Department of Electrical Engineering and Information Technology, Universitas Gadjah Mada, Yogyakarta 55281, Indonesia (e-mail: lukito@ugm.ac.id). He obtained his M.Sc. degree from James Cook University and his Ph.D. from Monash University, both in Australia. His research interests include mobile and pervasive computing, software engineering, and application of ICT in education.

Submitted 04 September 2015. Published as resubmitted by the authors 26 December 2015. 